International Journal of Case Reports
(ISSN:2572-8776)

\title{
CARDIAC METASTATIC INVOLVEMENT IN LUNG CANCER - CARDIAC METASTATIC INVOLVEMENT
}

Miguel Borregón ${ }^{1^{*}}$, Katherine Martínez ${ }^{2}$, Alba Ramos $^{1}$, Irene Ramos ${ }^{1}$, Beatriz Berzal ${ }^{1}$, Manuel Mazariegos ${ }^{1}$, Margarita Díez ${ }^{1}$, Cristina Gómez ${ }^{1}$, Ana Belén Rupérez¹, Irene Otero, Ana Karina Santos ${ }^{1}$, Juan David Cárdenas ${ }^{1}$, Elia Martínez ${ }^{3}$

${ }^{1}$ Medical oncology service. Hospital Virgen de la Salud de Toledo, Spain; ${ }^{2}$ Medical oncology service. Hospital General de Segovia, Spain; ${ }^{3}$ Medical oncology service. Hospital Universitario de Fuenlabrada, Spain.

\section{ABSTRACT}

Lung cancer is the second most common solid tumour and the first in mortality worldwide. Important progress has been reached in recent years in this pathology, focusing its treatment on histology, molecular biology and clinical presentation. Cardiac metastatic involvement consists in a severe clinical entity with increasing incidence due to better radiological techniques and longer survival of lung cancer. It requires special care and attention from many specialists. Clinical review about cardiac involvement secondary to lung cancer is provided, focused on its epidemiology, clinical presentation, diagnoses and treatment. Better understanding about cancer natural history is needed to offer the best treatment and care.

\section{Condensed abstract}

Cardiac metastatic involvement consists in a severe clinical entity with increasing incidence due to better radiological techniques and longer survival of lung cancer. It requires special care and attention from many specialists. Clinical review about cardiac involvement secondary to lung cancer is provided, focused on its epidemiology, clinical presentation, diagnoses and treatment.

Keywords: Lung cancer, Heart metastases, Malignant pericardial effusion, Cardiac tamponade

*Correspondence to Author:

Miguel Borregón

Complete postal address: Calle Fuenterrabía $n^{\circ} 4$ piso $6^{\circ}$ centro izquierda, Madrid, Madrid, Spain, 28014.

Telephone: 0034680870117.

How to cite this article:

Miguel Borregón, Katherine Martínez, Alba Ramos, Irene Ramos, Beatriz Berzal, Manuel Mazariegos, Margarita Díez, Cristina Gómez, Ana Belén Rupérez, Irene Otero, Ana Karina Santos, Juan David Cárdenas, Elia Martínez. CARDIAC METASTATIC INVOLVEMENT IN LUNG CANCER - CARDIAC METASTATIC INVOLVEMENT. International Journal of Case Reports, 2021, 5:252.

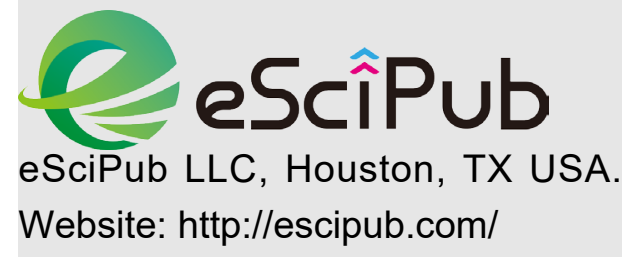


Lung cancer represents the second solid tumour in incidence and the first in mortality worldwide. It encompasses a wide spectrum of pathology with significant histological, molecular, and clinical variability and treatment options. This pathology has experienced a significant improvement in recent years thanks to therapeutic advances by the hands of targeted therapies and immunotherapy guided by better understanding of molecular biology and tumour microenvironment. Together with important prognostic factors as histology, clinical presentation and staging, best treatment option is selected for each patient. Lung cancer dissemination to cardiac tissue generally occurs in advanced stages coexisting with metastases in other locations. Lymphatic route is the most frequent way of spreading to this site ${ }^{[1,2]}$.

Metastatic cardiac involvement involves a wide spectrum of clinical presentation, from the absence of symptoms to life-threatening disease. It is an infrequent anatomy location, but its incidence is increasing in recent years due to radiological techniques development, including echocardiography, computed tomography (CT) scan, positron emission tomography (PET) and cardiac magnetic resonance imaging (MRI), and related to increased survival of cancer patients. Lung cancer is its main source. Metastatic cardiac involvement is more frequent than primary tumours, constituting around $95 \%$ of cardiac malignant involvement. Primary tumours are rare, being less than $10 \%$ diagnosed given their usually asymptomatic behaviour. Most are benign, being myxoma the most frequent histology. Sarcomas are the leading cause of malignant histology ${ }^{[3,4]}$.

Metastases in lung cancer appear, in order of frequency, in central nervous system (47\%), bone $(36 \%)$, liver $(22 \%)$, adrenal gland (15\%), thorax $(11 \%)$ and extra-thoracic lymph nodes $(10 \%)$. Metastases incidence in other anatomic locations is low, representing around $5 \%$ of all metastases, and they are often underdiagnosed. The theory that explains the low frequency of these metastatic sites suggests that some organs are somehow protected from metastatic seeding, being postulated tumour microenvironment as the fundamental regulatory condition. Microenvironment of tissues where metastases are rare, such as skin, spleen, kidney, soft tissues, gut and heart, is considered to express unfavourable characteristics for the anchoring and proliferation of primitive metastatic cells. Some of these characteristics are: presence of many leukocytes, scarce vascularization and large variations in blood supply and $\mathrm{pH}$. Under these physiological conditions, primitive metastatic cells are not likely to get clinically significant size [5-8].

Fei-Yu Niu and his team conducted a retrospective study of 2872 patients diagnosed with lung cancer, approaching to infrequent metastasis locations. They found a global incidence of $6.7 \%$ for these uncommon metastases, being adenocarcinoma the most frequent histology of primary tumour, with a large difference with respect to squamous cell carcinoma. The most affected organs were: soft tissues $(2.86 \%)$, kidney $(0.87 \%)$, peritoneum $(0.84 \%)$, spleen $(0.66 \%)$, pancreas $(0.59 \%)$, cardiac tissue $(0.07 \%)$. According to the anatomic metastatic location, statistically significant differences in overall survival were found, being lower in infrequent locations compared to common locations (13.0 versus 5.9 
months) [9].

Additionally, Shosaku and his team analysed cardiac involvement in lung cancer patient's autopsies, finding that it appeared in $44.4 \%$ of patients. The most frequent histological subtype was adenocarcinoma $(59.70 \%)$, followed by microcytic carcinoma (17.91\%) and squamous cell carcinoma (16.42\%). Most of them had been clinically irrelevant and undiagnosed during life [10].

The heart anatomic location of cardiac metastases has also been reviewed, finding that $65.8 \%$ take place in the pericardium (generally attributed to retrograde lymphatic spread), $29.0 \%$ in the myocardium, $24.9 \%$ in the epicardium, and $3.1 \%$ in the endocardium (attributed to uncommon hematogenous spread) ${ }^{[11,12]}$.

Clinical course of cardiac involvement is generally asymptomatic. When this affectation is diagnosed, symptoms are present in approximately $10 \%$ of patients. Clinical problems usually derive from pericardial effusion, being less frequent ischemic heart disease (due to tumour compression of coronary arteries) or arrhythmias. The most common symptoms are dyspnoea, cough, palpitations and chest pain. Hemodynamic instability (hypotension and tachycardia) can also be observed secondary to obstruction of ventricular output by the tumour mass itself or pericardial effusion. Important too, compared to non-malignant aetiologies, malignant pericardial effusion leads to higher rates of tamponade. According to the TNM classification developed by the American Joint Committee on Cancer (AJCC) it is classified as metastatic M1a and stage IV [13-16].

It is believed that malignant cardiac involvement, when diagnosed, is always associated with poor prognosis. There are studies that point in favour and against this assumption. Some clinical studies found an overall survival reduction in lung cancer patients suffering pericardial effusion with respect to patients who do not present it (7.6 versus 15.0 months). Likewise, it has been described a mortality increase in these patients with respect to patients presenting metastases in other locations also classified as M1a (malignant pleural effusion and contralateral pulmonary nodules) and with respect to metastases in other anatomical regions outside the thorax, classified as $\mathrm{M} 1 \mathrm{~b}$. On the other hand, negative studies have also been published. Liu Y.C. and his team analysed patients with metastatic hepatocarcinoma with and without cardiac involvement, finding no significant differences in overall survival, and negative implications only in patients suffering hemodynamic compromise. From the literature it is revealed that cardiac affection usually appears in patients with polytopic metastatic involvement, advanced stage disease and general condition deterioration, factors associated to decreased survival. Diagnostic delay related to lack of specificity of its symptoms can also be a reason connected to this hypothetical survival compromise [17-21].

Metastatic cardiac involvement is sometimes diagnosed incidentally by routine CT scan for extension evaluation of the disease. However, the gold-standard diagnostic test for its diagnosis is cardiac echocardiography. Cardiac MRI has also supposed a great advance in its identification and categorization. Chest radiography and electrocardiogram usefulness must be emphasized too. These diagnostic methods are very accessible and easy to interpret. Alterations in the S-T and Q-S complexes have been found to be the most 
frequent, but with little sensitivity and specificity. Classic semiology of cardiac tamponade on radiography is cardiomegaly, and on electrocardiogram, electrical alternation and QRS complex amplitude decrease might be found. These data, together with suggestive clinical features (tachycardia, dyspnoea, jugular ingurgitation and hemodynamic shock), collect very high sensitivity leading to diagnostic suspicion. Cardiac tamponade comprises an oncologic emergency that must be ruled out in every oncologic patient presenting these symptoms, higher probability if primary tumour is lung cancer [22, 23].

Certain diagnosis of metastatic cardiac affectation is histological. However, cardiac biopsy often involves more risk than benefit, given its compromised anatomical location and its limited implication in the therapeutic approach of the patient. On the other hand, cytological analysis of pericardial fluid offers high sensitivity if large effusion is drained. This can even be the analysis that confirms diagnosis of a new case of lung cancer. When pericardial effusion is not significant enough to justify its drainage as therapy, cytological analysis is not usually performed due to pericardiocentesis associated risks. In these cases, diagnosis of cardiac involvement becomes an exclusion diagnosis. It will be established on the basis of the existence of advanced primary tumour, the presence of cardiac mass or pericardial effusion, and the absence of alternative diagnosis.

Regarding cardiac metastases treatment, there is no standard algorithm or indications, and few studies have been published, generally case series. Treatment to be chosen depends on multiple factors, mainly histology, molecular biology, tumour extension, comorbidity, symptomatology and patient general condition. Surgical and radiotherapeutic treatment may be indicated in scenarios such as blood flow obstruction or cardiac metastatic involvement as the only metastatic site. Cardiac radiotherapy can be indicated when rapid response is needed or when other treatments are contraindicated. It can associate severe toxicity such as pericarditis, arrhythmias, cardiac fibrosis, and coronary or valvular disease ${ }^{[24]}$. Systemic therapy is the key treatment in advanced stages of lung cancer. At this moment, cardiac involvement by itself does not drive the best drug approach for each patient. Metastatic cardiac involvement in lung cancer consists in a rare and heterogeneous clinical presentation, with great diversity in histology, molecular biology, clinical features and response to treatment. Targeted therapies and immunotherapy, together with cytotoxic chemotherapy, have opened up a therapeutic and prognostic horizon that was difficult to picture just a few years ago. We are witnessing a paradigm shift in a disease of extreme severity and poor prognosis, leading cause of cancer mortality worldwide, in which the therapeutic possibilities have vastly been expanded in recent years. Understanding natural history and behaviour of these tumours and their metastatic involvement represents fundamental basis for optimizing their clinical management.

\section{Perspective}

The main aim of this manuscript is to describe and review the state of the art of cardiac metastatic involvement, focusing on its main tumour site of origin, lung cancer.

This manuscript reviews published literature to date focusing in epidemiology, clinical implication, diagnostic and therapy in this threatening metastatic affectation. 
Better understanding of the natural history and behaviour of tumours is a fundamental basis for better clinical management and oncology development.

\section{Disclosure statement}

The authors of this article declare that there is no conflict of interest with respect to its publication. All the authors have participated in the study and have read and approved the manuscript. Likewise, we assume all the responsibility about it contents.

\section{Funding source}

The authors of this article declare that there is no funding source for this study other than the base salary derived from the work in the public Spanish health system of its participants.

\section{Acknowledgements}

The authors of this manuscript acknowledge to Dr. Jose Ignacio Chacón, Medical Oncologist of Medical Oncology Department in Hospital Virgen de la Salud, his contribution on the education of new oncologist

residents. He has always been open to help and guide clinical and researching projects.

We also would like to acknowledge to Dr. Elia Martínez. She is the light leading this review and many other researching activities and on-going projects.

\section{References}

[1] Burazor I, Aviel-Ronen S, Imazio M, et al. Metastatic cardiac tumours: from clinical presentation through diagnosis to treatment. BMC Cancer 2018; 18: 202.

[2] Meddeb M, Chow RD, Whipps R, et al. The heart as a site of metastasis of benign metastasizing leiomyoma: case report and review of the literature. Case Rep Cardiol 2018; 2018: 7231326.

[3] Mercado-Guzman MP, Meléndez-Ramírez G, Castillo-Castellon $\mathrm{F}$, et al. Evaluation of cardiac tumours by multidetector computed tomography and magnetic resonance imaging. Arch Cardiol Mex 2016; 86: 33549.

[4] Nomoto N, Tani T, Konda T, et al. Primary and metastatic cardiac tumours: echocardiographic diagnosis, treatment and prognosis in a 15-years single centre study. J Cardiothorac Surg 2017; 12: 103.

[5] Perisano C, Spinelli MS, Graci C, Scaramuzzo L, Marzetti E, Barone C, Fabbriciani C. Soft tissue metastases in lung cancer: a review of the literature. Eur Rev Med Pharmacol Sci. 2012;16(14):1908-14.

[6] Scatena C, Comin CE, Lapini A, Raspollini MR. Renal metastasis from pulmonary adenocarcinoma: the pathologist's approach to an uncommon finding: case report and review of the literature. Appl Immunohistochem Mol Morphol. 2013;21(5):460-3.

[7] Kovacs KA, Hegedus B, Kenessey I, Timar J. Tumor type-specific and skin region-selective metastasis of human cancers: another example of the "seed and soil" hypothesis. Cancer Metastasis Rev. 2013;32(34):493-9.

[8] Plaza JA, Perez-Montiel D, Mayerson J, Morrison C, Suster S. Metastases to soft tissue: a review of 118 cases over a 30-year period. Cancer. 2008;112(1): 193-203.

[9] Fei-Yu Niu, Qing Zhou, Jin-Ji Yang, Wen-Zhao Zhong, Zhi-Hong Chen, Wei Deng, Yan-Yan He, Hua-Jun Chen, Zhu Zeng, E-E Ke, Ning Zhao, Na Zhang, Hui-Wen Sun, Qiu-Yi Zhang, Zhi Xie, XuChao Zhang and Yi-Long Wu; Distribution and prognosis of uncommon metastases from non-small cell lung cancer; Niu et al. BMC Cancer (2016) 16:149; DOI 10.1186/s12885-016-2169-5.

[10] Shosaku A.B.E., Naomi Watanabe, Shigeaki Ogura, Hiroshi Kunikane, Hiroshi Isobe, Etsuro Yamaguchi, Mitsuru Munakata and Yoshikazu Kawakami; Myocardial Metastasis from Primary Lung Cancer: Myocardial Infarction-like ECG Changes and Pathologic Findings; Jpn J Med Vol 30, No 3 (May, June 1991). 
[11] Butany J., Nair V., Naseemuddin A., Nair G.M., Catton C., Yau T.; Cardiac tumours: diagnosis and management; Lancet Oncology 6:219-228, 2006.

[12] Reynen K., Kockeritz U., Strasser R.H.; Metastases to the heart; Ann Oncol 2004; 15:375-81.

[13]Zakharova VP, Rudenko YeV, Galakhin KA, et al. Cardiac mixomas (morphological aspects). Kyiv: Kniga-plyus; 2003. 142 p.

[14] Tsang TS, Seward JB, Barnes ME, Bailey KR, Sinak LJ, Urban LH, et al. Outcomes of primary and secondary treatment of pericardial effusion in patients with malignancy. Mayo Clin Proc. 2000 75(3);248253. https://doi.org/10.4065/75.3.248.

[15] Gornik HL, Gerhard-Herman M, Beckman JA. Abnormal cytology predicts poor prognosis in cancer patients with pericardial effusion. J Clin Oncol.2005(22); 23:5211-5216. https://doi.org/10.1200/JCO. 2005.00.745.

[16] Kim SH, Kwak MH, Park S, Kim HJ, Lee HS, Kim MS, et al. Clinical characteristics of malignant pericardial effusion associated with recurrence and survival. Cancer Res Treat.2010; 42 (4):210-216. https:// doi.org/10.4143/crt.2010.42.4.210 PMID: 21253323.

[17] Ou SH, Zell JA. Validation study of the proposed IASLC staging revisions of the T4 and M non-small cell lung cancer descriptors using data from 23,583 patients in the California Cancer Registry. J Thorac Oncol.2008; 3(3): 216-227. https://doi.org/10.1097/JTO.0b013e318164545d PMID: 18317063.

[18] Kato R, Hayashi H, Chiba Y, Tanaka K, Takeda M, Nakagawa K. Prognostic impact of minimal pericardial effusion in patients with advanced Non-small-cell Lung Cancer. Clin Lung Cancer. 2017; 18(6): e449e455. https://doi.org/10.1016/j.cllc.2017.05.011.

[19] Hoffmeier A, Sindermann JR, Scheld HH, et al. Cardiac tumours - diagnosis and surgical treatment. Dtsch Arztebl Int 2014; 111: 205-11.
[20] Pacini D, Careddu L, Pantaleo A, et al. Primary malignant tumours of the heart: Outcomes of the surgical treatment. Asian Cardiovasc Thorac Ann 2015; 23: 645-51.

[21] Liu Y.C.; Ho Y.L., Huang G.T., Chen D.S., Sheu J.C., Chen C.H.; Clinical manifestation and survival of patients with hepatocelular carcinoma and cardiac metastases; J. Gastroenterol Hepatol (2010) 25:150-5.

[22] Deetjen A., Conradi G., Mollmann S., Kluge A., Hamm C.W., Dill T; Cardiac metastases of a renal cell adenocarcinoma investigated by cardiac magnetic resonance imaging; Clin Res Cardiol 2006;95:492-5.

[23] Cates C.U., Virmani R., Vaughn W.K., Robertson R.M.; Electrocardiographic markers of cardiac metastases; Am. Heart J. 112: 1297, 1986.

[24] Chiu-Fan Chen, Min-Hsi Lin, Kuo-An Chu, Wen-Shan Liu, Shih-Hung Hsiao, Ruay-Sheng Lai; Effective cardiac radiotherapy relieved life-threatening heart failure caused by advanced small cell lung cancer with cardiac metastasis: a case report; Journal of thoracic disease, 2018; 10(4): E250-E254.

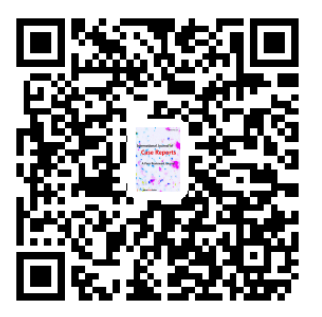

